\title{
CHARACTER TABLES OF METACYCLIC GROUPS
}

\author{
STEPHEN P. HUMPHRIES AND DANE C. SKABELUND \\ Department of Mathematics, Brigham Young University, Provo, UT 84602, U.S.A. \\ e-mail: steve@math.byu.edu,dane.skabelund@gmail.com
}

(Received 4 June 2013; accepted 8 April 2014; first published online 17 December 2014)

\begin{abstract}
We show that two metacyclic groups of the following types are isomorphic if they have the same character tables: (i) split metacyclic groups, (ii) the metacyclic $p$-groups and (iii) the metacyclic $\{p, q\}$-groups, where $p, q$ are odd primes.

2010 Mathematics Subject Classification. Primary 20C15; Secondary 20D15, 20F16, 20F22.
\end{abstract}

1. Introduction. A group $G$ is metacyclic if it has a cyclic normal subgroup $K$ such that $G / K$ is cyclic. Equivalently, the group $G$ has cyclic subgroups $S$ and $K$, with $K$ normal in $G$, such that $G=S K$. If we can find such $H, K$ with $H \cap K=\{1\}$, then we say that $G$ is split metacyclic. It was shown by Hölder [11, Theorem 7.21] that a group is metacyclic if and only if it has a presentation of the form

$$
G=G_{\alpha, \beta, \gamma, \delta}=\left\langle a, b \mid a^{\alpha}=b^{\delta}, b^{\beta}=1, b^{a}=b^{\gamma}\right\rangle,
$$

where $\operatorname{gcd}(\beta, \gamma)=1, \beta \mid\left(\gamma^{\alpha}-1\right)$ and $\beta \mid \delta(\gamma-1)$. However, in general the parameters $\alpha, \beta, \gamma$, and $\delta$ are not invariants of the group $G$. Note that if $\delta=0$, then $G$ is split metacyclic.

One says that two groups $G, G^{\prime}$ have the same character tables if there is a bijection $\chi \leftrightarrow \chi^{\prime}$ of their irreducible characters and a bijection $(g)^{G} \leftrightarrow\left(g^{\prime}\right)^{G^{\prime}}$ of their classes such that $\chi(g)=\chi^{\prime}\left(g^{\prime}\right)$. This amounts to saying that their character tables are the same up to permuting rows and columns.

In this paper, we are interested in the question of when a metacyclic group is determined by its character table (see [2] for a nice account of the character table). This is not always the case, as, for example, the quaternion and dihedral groups $Q_{8}=$ $G_{2,4,3,2}, D_{8}=G_{2,4,3,0}$ of order 8 are metacyclic and have the same character table. Note that here $D_{8}$ is split metacyclic, while $Q_{8}$ is not. We prove:

THEOREM 1.1. Any two split metacyclic groups with the same character tables are isomorphic.

More generally, it is well-known that the dihedral group $D_{2^{n}}$ and the generalized quaternion group $Q_{2^{n}}$ of order $2^{n}, n \geq 3$, have the same character tables [4, p.64]. Presentations for these groups are

$$
D_{2^{n}}=G_{2,2^{n-1},-1,0} ; \quad Q_{2^{n}}=G_{2,2^{n-1},-1,2^{n-2}} .
$$

We prove: 
THEOREM 1.2. Among metacyclic p-groups, the only pairs having the same character tables are $D_{2^{n}}$ and $Q_{2^{n}}, n \geq 3$.

We conjecture that no two non-isomorphic metacyclic groups of odd order have the same character tables. In further support of this we show:

TheOREM 1.3. Any two metacyclic $\{p, q\}$-groups, $p, q$ odd, can be distinguished by their character tables.

Here, for primes $p, q$, a $\{p, q\}$-group is a group whose only prime divisors are the primes $p, q$.

These three results are proved in the next three sections. All groups are assumed to be finite.

2. Split metabelian groups. We first note the following facts about metacyclic groups: subgroups and quotients of metacyclic groups are metacyclic, however subgroups and quotients of split metacyclic groups need not be split metacyclic. A metacyclic group may have the same character table as a non-metacyclic group (e.g. extra-special $p$-groups).

The ambiguity of the choice of parameters $\alpha, \beta, \gamma, \delta$ for $G_{\alpha, \beta, \gamma, \delta}$ is exemplified by the isomorphism $G_{6,36,19,0} \cong G_{18,12,7,0}$ of split metacyclic groups. This isomorphism arises from the fact that the Sylow three-subgroup has the form $\mathcal{C}_{3} \times \mathcal{C}_{9}$ (where $\mathcal{C}_{n}$ is the cyclic group of order $n$ ) and is a direct factor of the metacyclic group. We address this difficulty in the next result:

LeMma 2.1. Let $G$ be a group with a central Sylow p-subgroup $P$. Then $G$ has $P$ as a direct factor and we write $G=P \times H$. Both the isomorphism type of $P$ and the character table of the complement $H$ are determined by the character table of $G$.

Proof. If $G$ has a central Sylow $p$-subgroup $P$, then $P$ is normal in $G$ and so it is the unique normal subgroup of its order. Thus, the the conjugacy classes comprising $P$ can be located using the character table of $G$. Since $P$ is central, and the multiplication table of $Z(G)$ is determined by the character table of $G$, from this we can determine the isomorphism type of $P$. Since $P$ is a normal Hall subgroup, the group $G$ splits over $P$ by the Schur-Zassenhaus theorem [7, p.75], and we write $G=P \rtimes H$ for some $H$. But since $P$ is central, this product is direct, and we have $G=P \times H$. The character table of the quotient $H=G / P$ can be obtained from the character table of $G$. Furthermore, since $G=P \times H$, and we know the isomorphism type of $P$, the isomorphism type of $G$ is determined by the isomorphism type of $H$.

Suppose that $G$ has a central Sylow $p$-subgroup $P$, and let $\alpha_{p}$ and $\beta_{p}$ be the powers of $p$ dividing $\alpha$ and $\beta$. Then $P=\left\langle a^{\alpha / \alpha_{p}}\right\rangle \times\left\langle b^{\beta / \beta_{p}}\right\rangle$, and so we have the presentation

$$
G / P \cong\left\langle a, b \mid a^{\alpha / \alpha_{p}}=b^{\beta / \beta_{p}}=1, b^{a}=b^{\gamma}\right\rangle,
$$

which shows that $G / P$ is split metacyclic. It follows from Lemma 2.1 that if we are able to determine the isomorphism type of $G / P$ from its character table, then we will be able to determine the isomorphism type of $G$ from its character table. From now on we assume that no Sylow $p$-subgroup of $G$ is central. We will need:

LEMMA 2.2. ([10, Lemma 2.7]) Let $G$ be a group with a metacyclic factorization $G=$ $S K$. Let $S=\langle a\rangle$ and $K=\langle b\rangle \unlhd G$. Let $\gamma$ be an integer with $b^{a}=b^{\gamma}$. Let $s=\operatorname{ord}_{|K|}(\gamma)$ 
and $t=|K| / \operatorname{gcd}(|K|, \gamma-1)$. Then

$$
G^{\prime}=\left\langle b^{\gamma-1}\right\rangle \cong \mathcal{C}_{t}, \quad Z(G)=\left\langle a^{s}, b^{t}\right\rangle
$$

By Lemma 2.2, we know that

$$
G^{\prime}=\left\langle b^{\gamma-1}\right\rangle \cong \mathcal{C}_{t}, \quad Z(G)=\left\langle a^{s}, b^{t}\right\rangle,
$$

where $s=\operatorname{ord}_{\beta}(\gamma)$ divides $\alpha$, and $t=\beta /(\beta, \gamma-1)$. Thus $t$ can be determined from the character table. Since $G$ is split, the subgroups generated by $a^{s}$ and $b^{t}$ intersect trivially, and so $Z(G)=\left\langle a^{s}\right\rangle \times\left\langle b^{t}\right\rangle \cong \mathcal{C}_{u} \times \mathcal{C}_{v}$, where

$$
u=\left|a^{s}\right|=\alpha / s \quad \text { and } \quad v=\left|b^{t}\right|=\beta / t .
$$

LEMMA 2.3. With the above assumption on central p-subgroups, the character table of $G_{\alpha, \beta, \gamma, 0}$ determines $\alpha, \beta, s, t, u$, and $v$.

Proof. So far we have been able to determine

$$
|G|=\alpha \beta, \quad|Z(G)|=u v, \quad\left|G^{\prime}\right|=t, \quad \text { and } \quad s=\frac{|G|}{|Z(G)| \cdot\left|G^{\prime}\right|} .
$$

We note that if at any time we are able to determine one of the parameters $\alpha, \beta, u$, or $v$, then we will be able to determine them all. Also, if we are able to determine the power of a prime $p$ which divides one of these, we can determine the power of $p$ which divides each of them.

We can determine from the character table the isomorphism types of the following abelian groups in terms of the parameters given above:

$$
\begin{aligned}
Z(G) & \cong \mathcal{C}_{u} \times \mathcal{C}_{v}, \\
G / G^{\prime} & \cong \mathcal{C}_{\alpha} \times \mathcal{C}_{v}, \\
Z /\left(G^{\prime} \cap Z(G)\right) & \cong \mathcal{C}_{u} \times \mathcal{C}_{v /(t, v)}
\end{aligned}
$$

Thus, we know the invariant factors of each, and in particular we can determine the power of $p$ which divides the order of each direct factor of these groups, up to the ordering of the factors. Let $p$ be a prime. For a positive integer $x$, we write $x_{p}$ to denote the power of $p$ that divides $x$. Then

$$
A_{p}=\left\{u_{p}, v_{p}\right\}, \quad B_{p}=\left\{\alpha_{p}, v_{p}\right\}, \quad \text { and } \quad C_{p}=\left\{u_{p}, v_{p} /\left(t_{p}, v_{p}\right)\right\},
$$

are the sets of invariant factors for the $p$-primary parts of the above groups, and these sets are determined by the character table.

If $\left|A_{p} \cap B_{p}\right|=1$, then $A_{p} \cap B_{p}=\left\{v_{p}\right\}$ and we are done. Thus, we assume that $\left|A_{p} \cap B_{p}\right|=2$, so that

$$
u_{p}=\alpha_{p} \neq v_{p}
$$

Next, if $\left|A_{p} \cap C_{p}\right|=1$, then $A_{p} \cap C_{p}=\left\{u_{p}\right\}$ and we are done. Thus, we also assume that $\left|A_{p} \cap C_{p}\right|=2$, so that

$$
v_{p}=v_{p} /\left(t_{p}, v_{p}\right) \text { and }\left(t_{p}, v_{p}\right)=1 .
$$


If $t_{p} \neq 1$, then we have $v_{p}=1$ and we are done. We now assume that $t_{p}=1$. But now $\beta_{p}=t_{p} v_{p}=v_{p}$, and so $(\alpha \beta)_{p}=\alpha_{p} \beta_{p}=u_{p} v_{p}=(u v)_{p}$. Therefore, a Sylow $p$-subgroup of $G$ is central, which contradicts our assumption.

We show how to determine $\gamma$, by first noting that it is sufficient to find the subgroup of $(\mathbb{Z} / \beta \mathbb{Z})^{\times}$generated by $\gamma$ :

LEMMA 2.4. ([1, Lemma 7]) If $G_{i}=G_{\alpha, \beta, \gamma_{i}, 0}, i=1,2$, are split metacyclic groups such that $\left\langle\gamma_{1}\right\rangle=\left\langle\gamma_{2}\right\rangle$ as subgroups of $(\mathbb{Z} / \beta \mathbb{Z})^{\times}$, then $G_{1}$ and $G_{2}$ are isomorphic.

There is a classical combinatorial method for constructing the irreducible representations of the symmetric groups $S_{n}$. This method makes use of Young tableau in order to construct elements of the group algebra $\mathbb{C} S_{n}$, called Young symmetrizers, which generate all the minimal ideals of $\mathbb{C} G$. These ideals correspond to the irreducible representations of $S_{n}$. For more information about this, see [5, Ch 4] or [3, Ch 28]. In [9], Munkholm uses similar methods to construct the irreducible representations of metacyclic groups. Here is a statement of this result.

THEOREM 2.5. [9, Theorem 5.1] Let $G=G_{\alpha, \beta, \gamma, \delta}$ be a metacyclic group where

$$
\operatorname{gcd}(\beta, \gamma)=1, \delta(\gamma-1) \equiv \gamma^{\alpha}-1 \equiv 0 \quad(\bmod \beta), \beta>0, \gamma>0, \alpha>0, \delta \geq 0 .
$$

Let $s_{i}$ be the order of $\gamma$ as an element of the group of units in $\mathbb{Z} /\left(\frac{\beta}{(\beta, i)}\right) \mathbb{Z}$. In $\{0,1, \ldots, \beta-$ 1) we define an equivalence relation $\sim b y: i \sim i^{\prime}$ if and only if there is a $v$ with $i \equiv i^{\prime} \gamma^{v}$ $(\bmod \beta)$, and we let $I$ be a set of representatives of the classes modulo $\sim$. For each $i \in I$ we let $\left\{q_{i, 1}, q_{i, 2}, \ldots, q_{i, \alpha / s_{i}}\right\}$ be a full set of pairwise incongruent integers modulo $\alpha / s_{i}$ with $-i \delta / \beta \leq q_{i, k}<\alpha-i \delta / \beta$, and we put

$$
j_{i, k}=i \delta /(\beta, \delta)+q_{i, k} \beta /(\beta, \delta) .
$$

Finally, we choose a primitive $\beta$ th root of unity $\zeta$ and a primitive nth root of unity $\eta$ $(n=\alpha \beta /(\beta, \delta))$ such that $\zeta^{(\beta, \delta)}=\eta^{\alpha}$. Then some of the Young-elements are:

$$
e_{i, j_{k, i}}=\sum_{m=0}^{\beta-1} \sum_{n=0}^{\alpha-1} \zeta^{m i} \eta^{n j_{i, k}} a^{m} b^{n}, \quad k=1,2, \ldots, \alpha / s_{i}, \quad i \in I,
$$

and

$$
\left\{(\mathbb{C} G) e_{i, j_{i, k}} ; \quad k=1,2, \ldots, \alpha / s_{i}, \quad i \in I\right\},
$$

is a full set of pairwise inequivalent, irreducible left $\mathbb{C} G$-modules. The representation $R_{i k}$ afforded by $(\mathbb{C} G) e_{i, j_{i, k}}$ is of degree $s_{i}$ and it is induced from the linear representation $T_{i k}: G_{i} \rightarrow \mathbb{C}$ where $G_{i}$ is the subgroup of $G$ generated by the elements $a^{s_{i}}$ and $b$, and where the action of $T_{i k}$ is given by the formulae:

$$
T_{i k}\left(a^{s_{i}}\right)=\eta^{s_{j} j_{i, k}}, \quad T_{i k}(b)=\zeta^{i} .
$$

Let

$$
\zeta=e^{2 \pi i / \beta}, \quad \eta=e^{2 \pi i / \alpha}, \quad L=\mathbb{Q}(\zeta) \text { and } H=\langle\gamma\rangle \leq(\mathbb{Z} / \beta \mathbb{Z})^{\times} .
$$


Since $(\mathbb{Z} / \beta \mathbb{Z})^{\times}=\operatorname{Gal}(L / \mathbb{Q})$, there is a Galois correspondence between the subgroups of $(\mathbb{Z} / \beta \mathbb{Z})^{\times}$and the subfields of $L$. Thus by Lemma 2.4 , we will be done if we are able to determine the fixed field $L^{H}$ from the character table of $G$.

We restate Theorem 2.5 specialized to the situation of a split metacyclic group. Let $I$ be a complete set of representatives of the orbits under the multiplicative action of $\langle\gamma\rangle$ on $\mathbb{Z} / \beta \mathbb{Z}$, and for each $i \in I$ let $s_{i}$ denote the order of $\gamma$ as an element of $\left(\mathbb{Z} / \frac{\beta}{(\beta, i)} \mathbb{Z}\right)^{\times}$. Then a complete set of inequivalent irreducible complex representations of $G$ is obtained by inducing certain linear representations $T_{i k}$ of the subgroups $G_{i}=\left\langle a^{s_{i}}, b\right\rangle$ to $G$. Explicitly, these representations of $G_{i}$ are given by the formulae

$$
T_{i k}\left(a^{s_{i}}\right)=\eta^{s_{i} k}, \quad T_{i k}(b)=\zeta^{i},
$$

where $0 \leq k<\alpha / s_{i}$. We induce the characters $T_{i k}$ to $G$ to obtain characters $\chi_{i, k}$ which vanish off of the (normal) subgroup $G_{i}=\left\langle a^{s_{i}}, b\right\rangle$. We use the transversal $1, a, \ldots, a^{s_{i}-1}$ for $G_{i}$ in $G$ to compute the values of $\chi_{i, k}$ as follows:

$$
\begin{aligned}
\chi_{i, k}\left(a^{s_{i} \sigma} b^{\tau}\right) & =\sum_{\ell=0}^{s_{i}-1} T_{i k}\left(\left(a^{s_{i} \sigma} b^{\tau}\right)^{a^{\ell}}\right)=\sum_{\ell=0}^{s_{i}-1} T_{i k}\left(a^{s_{i} \sigma} b^{\tau \gamma^{\ell}}\right) \\
& =\sum_{\ell=0}^{s_{i}-1} \eta^{s_{i} \sigma k} \zeta^{i \tau \gamma^{\ell}}=\eta^{s_{i} \sigma k} \sum_{\ell=0}^{s_{i}-1} \zeta^{i \tau \gamma^{\ell}}
\end{aligned}
$$

From now on, we will only need to consider the characters $\chi_{i, k}$ of maximal degree $s$, so when we refer to "the $\chi_{i, k}$ " we will only mean these. These characters are those with $s_{i}=s$, and they take the values

$$
\chi_{i, k}\left(a^{s \sigma} b^{\tau}\right)=\eta^{s \sigma k} \sum_{\ell=1}^{s} \zeta^{i \tau \gamma^{\ell}}
$$

For $i \in I$, let

$$
\xi_{i}=\operatorname{Tr}_{L^{H}}^{L}\left(\zeta^{i}\right)=\sum_{\ell=1}^{s} \zeta^{i \gamma^{\ell}}
$$

We will call these periods, as they are the same as Gaussian periods when $i \in(\mathbb{Z} / \beta \mathbb{Z})^{\times}$. As traces over $L^{H}$ these periods are contained in $L^{H}$. More importantly, they generate the whole fixed field $L^{H}$, as is proved in the following lemma.

LemMA 2.6. Let $L / K$ be a finite extension of fields with basis $B$, and let $H$ be a subgroup of $\operatorname{Aut}(L / K)$. For $\lambda \in L$, let $\mathcal{C}_{\lambda}$ denote the orbit of $\lambda$ under the action of $H$, and let $\xi_{\lambda}=\sum_{\mu \in \mathcal{C}_{\lambda}} \mu$ (so that the periods $\xi_{i}$ above are $\xi_{\zeta^{i}}$ ). Then the set $\left\{\xi_{\lambda}: \lambda \in B\right\}$ spans $L^{H}$ as a $K$-vector space.

Proof. For $\lambda \in L$, let $\xi_{\lambda}^{\prime}=\sum_{\sigma \in H} \lambda^{\sigma}$. Then $\xi_{\lambda}^{\prime}$ is a positive integer multiple of $\xi_{\lambda}$, and so any set of $\xi_{\lambda}$ span the same subspace of $L$ as the corresponding set of $\xi_{\lambda}^{\prime}$. Let $\lambda \in L^{H}$, and write $\lambda=\sum_{\mu \in B} c_{\lambda, \mu} \mu$ with $c_{\lambda, \mu} \in K$. Then for any $\sigma \in H$, we have 
$\lambda=\lambda^{\sigma}=\sum_{\mu \in B} c_{\lambda, \mu} \mu^{\sigma}$. It follows that

$$
\lambda=\frac{1}{|H|} \sum_{\sigma \in H} \lambda^{\sigma}=\frac{1}{|H|} \sum_{\sigma \in H} \sum_{\mu \in B} c_{\lambda, \mu} \mu^{\sigma}=\frac{1}{|H|} \sum_{\mu \in B} c_{\lambda, \mu} \sum_{\sigma \in H} \mu^{\sigma}=\frac{1}{|H|} \sum_{\mu \in B} c_{\lambda, \mu} \xi_{\mu}^{\prime},
$$

and so $L^{H}$ is spanned by the $\xi_{\mu}^{\prime}, \mu \in B$.

COROLlaRY 2.7. The periods $\xi_{i}, i \in I$, span $\mathbb{Q}(\zeta)^{H}$.

Let $L_{i, k}$ be the field obtained by adjoining the values of $\chi_{i, k}$ to the rational field. Then from equation (2.1), we have $L_{i, k}=L_{i} N_{k}$, where

$$
N_{k}=\mathbb{Q}\left(\eta^{s k}\right) \text { and } L_{i}=\mathbb{Q}\left(\left\{\xi_{i \tau}: 0 \leq \tau \leq \beta-1\right\}\right) .
$$

For $i$ with $\operatorname{gcd}(i, \beta)=1$, the values $\xi_{i \tau}$ run through all the periods $\xi_{j}$ as $\tau$ runs through $0,1, \ldots, \beta-1$, and so $L_{i}=L^{H}$ by Corollary 2.7 .

By examining the values of the $\chi_{i, k}$ on $Z(G)$ we will be able to choose certain $\chi_{i, k}$ which will allow us to isolate the periods $\xi_{i}$ and determine the fixed field $L^{H}$. The values of $\chi_{i, k}$ on $Z(G)=\left\langle a^{s}\right\rangle \times\left\langle b^{t}\right\rangle \cong \mathcal{C}_{u} \times \mathcal{C}_{v}$ are

$$
\chi_{i, k}\left(a^{s \sigma} b^{t \tau}\right)=s \eta^{s \sigma k} \zeta^{t \tau i}, \quad 0 \leq \sigma \leq u, \quad 0 \leq \tau \leq v .
$$

Let $Y$ be the set of all $\chi_{i, k}$ such that $\chi_{i, k}(z) \in \mathbb{Q}\left(\zeta^{t}\right)$ for all $z \in Z(G)$. Since $\mathbb{Q}\left(\zeta^{t}\right)$ is the $v$ th cyclotomic field, we know which field $\mathbb{Q}\left(\zeta^{t}\right)$ is. Note that the fact that a character $\chi_{i, k}$ belongs to $Y$ puts no restraint on $i$, but only on $k$. In particular, the character $\chi_{i, 0}$ for any $i$ relatively prime to $\beta$ is contained in $Y$. As a result, the field obtained by adjoining to $\mathbb{Q}$ the set

$$
\left\{\chi_{i, k}(g): \chi_{i, k} \in Y, g \in G\right\}
$$

is equal to $\mathbb{Q}\left(\zeta^{t},\left\{\xi_{i \tau}\right\}_{i, \tau}\right)$. Since $\zeta^{t} \in L^{H}$ and the $\xi_{i \tau}$ generate $L^{H}$, this field is the desired fixed field. This completes the proof.

We give an example of the process described in the above proof of Theorem 1.1 for determining the isomorphism type of a split metacyclic group.

EXAMPLE 1. We consider group $G=G_{72,27}$ in Magma notation [8]. This group has 24 linear characters and 12 characters of degree 2 . We find the values

$$
\alpha \beta=|G|=72, \quad t=\left|G^{\prime}\right|=3 \text {, and } s=\max c . d .(G)=2,
$$

where $c . d .(G)$ denotes the set of character degrees of $G$. We can also determine the isomorphism types of

$$
\begin{aligned}
Z(G) & \cong \mathcal{C}_{u} \times \mathcal{C}_{v} \cong \mathcal{C}_{12}, \\
G / G^{\prime} & \cong \mathcal{C}_{\alpha} \times \mathcal{C}_{v} \cong \mathcal{C}_{2} \times \mathcal{C}_{12}, \\
Z /\left(G^{\prime} \cap Z(G)\right) & \cong \mathcal{C}_{u} \times \mathcal{C}_{v /(t, v)} \cong \mathcal{C}_{12}
\end{aligned}
$$

Then $A_{2}=\left\{u_{2}, v_{2}\right\}=\{1,4\}$ and $B_{2}=\left\{\alpha_{2}, v_{2}\right\}=\{2,4\}$. Since $A_{2} \cap B_{2}=\{4\}$, we have $v_{2}=4$. From this we determine that $\beta_{2}=t_{2} v_{2}=4, \alpha_{2}=(\alpha \beta)_{2} / \beta_{2}=2$, and $u_{2}=$ $\alpha_{2} / s_{2}=1$. We also have $A_{3}=\left\{u_{3}, v_{3}\right\}=\{1,3\}$ and $C_{3}=\left\{u_{3}, v_{3} /\left(3, v_{p}\right)\right\}=\{1,3\}$. Since these sets are the same, we must have $v_{3}=1$. From this we determine that $\beta_{3}=t_{3} v_{3}=3$, 
$\alpha_{3}=(\alpha \beta)_{3} / \beta_{3}=3$, and $u_{3}=\alpha_{3} / s_{3}=3$. Putting this information together, we have $(\alpha, \beta, s, t, u, v)=(6,12,2,3,3,4)$.

For the remaining steps, we will refer to the partial character table for $G$ given in Table 1 . This table gives the values of the 12 characters of $G$ of maximal degree 2 . The central classes are labelled with asterisks across the top. The characters which take on values on $Z(G)$ which are in $\mathbb{Q}(i)$ are characters $\chi_{1}, \chi_{2}, \chi_{7}$, and $\chi_{8}$. Adjoining the values of these characters gives the field $\mathbb{Q}(i)$. Since the subgroup of $(\mathbb{Z} / 12 \mathbb{Z})^{\times}$fixing $\mathbb{Q}(i)$ is the subgroup generated by 5 , we conclude that

$$
G=\left\langle a, b \mid a^{6}=b^{12}=1, b^{a}=b^{5}\right\rangle .
$$

3. $p$-groups. In this section, we prove Theorem 1.2.

We will need the following lemma, which is Corollary 4.4 of [6].

LEMMA 3.1. If $m \geq n \geq 1, p$ is a prime, $n+p \geq 4$, and $r \geq 1$, then each of the statements

$$
\begin{aligned}
\left(1+p^{n}\right)^{p^{r}} & \equiv 1 \bmod p^{m}, \\
\left(-1+2^{n}\right)^{2^{r}} & \equiv 1 \bmod 2^{m}
\end{aligned}
$$

is equivalent to $r \geq m-n$.

We first consider the $p$ odd case.

Let $G$ be a noncyclic metacyclic $p$-group, $p$ odd. Then in Theorem 3.5 of [10], it is shown that $G$ has a presentation of the form

$$
G=\left\langle a, b \mid a^{p^{\alpha}}=b^{p^{\beta}}, b^{p^{\beta+\delta}}=1, b^{a}=b^{p^{1+p^{\gamma}}}\right\rangle,
$$

where $\alpha, \beta, \gamma, \delta$ are nonnegative integers satisfying $\alpha \geq \beta \geq \gamma \geq \delta$ and $\gamma \geq 1$, and that these parameters characterize the group $G$.

From the character table, we can determine $|G|=p^{\alpha+\beta+\delta}$ and $G / G^{\prime} \cong \mathcal{C}_{p^{\alpha}} \times \mathcal{C}_{p^{\gamma}}$. Since $\alpha \geq \gamma$, we then know $\alpha$ and $\gamma$, and also $\beta+\delta$. We now determine the isomorphism type of $Z(G)$. From Lemma 2.2 we know that $Z(G)=\left\langle a^{s}, b^{t}\right\rangle$, where

$$
\begin{aligned}
& s=\operatorname{ord}_{|b|}\left(1+p^{\gamma}\right)=\operatorname{ord}_{p^{\beta+\delta}}\left(1+p^{\gamma}\right), \\
& t=|b| / \operatorname{gcd}\left(|b|, p^{\gamma}\right)=p^{\beta+\delta} / \operatorname{gcd}\left(p^{\beta+\delta}, p^{\gamma}\right)=p^{\beta+\delta-\gamma},
\end{aligned}
$$

and this last equality follows since $\beta+\delta \geq \gamma$. Since $\beta+\delta \geq \gamma \geq 1$ and $\gamma+p \geq 4$, Lemma 3.1 implies that $\left(1+p^{\gamma}\right)^{p^{\epsilon}} \equiv 1 \bmod p^{\beta+\delta}$ if and only if $\epsilon \geq \beta+\delta-\gamma$, and so

$$
s=\operatorname{ord}_{p^{\beta+\delta}}\left(1+p^{\gamma}\right)=p^{\beta+\delta-\gamma} .
$$

Since $|a|=p^{\alpha+\delta}$ and $|b|=p^{\beta+\delta}$, we have

$$
\begin{aligned}
& \left|a^{s}\right|=|a| / s=p^{\alpha+\delta} / p^{\beta+\delta-\gamma}=p^{\alpha-\beta+\gamma}, \\
& \left|b^{t}\right|=|b| / t=p^{\beta+\delta} / p^{\beta+\delta-\gamma}=p^{\gamma} .
\end{aligned}
$$


Table 1. Nonlinear characters of group $G_{72,27}$. Here $\omega=e^{2 \pi i / 3}, i=e^{2 \pi i / 4}, \zeta=e^{2 \pi i / 6}$, and $\eta=e^{2 \pi i / 12}$.

\begin{tabular}{|c|c|c|c|c|c|c|c|c|c|c|c|c|c|c|c|c|c|c|c|c|c|c|c|c|c|c|c|c|c|c|c|c|c|}
\hline & $*$ & * & & & $*$ & $*$ & & & & $*$ & * & & & $*$ & $*$ & & & & & & & $*$ & $*$ & $*$ & * & & & & & & & & \\
\hline & 2 & & 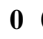 & & 2 & 2 & & & & 2 & 2 & & 0 & 2 & & - & & & & $\begin{array}{ll}0 & 0\end{array}$ & & & 2 & 2 & & -1 & -1 & 1 & & & $\begin{array}{ll}-1 & 0\end{array}$ & 0 & \\
\hline & 2 & 2 & 0 & & 2 & 2 & & & -1 & -2 & -2 & $\mathbf{0}$ & $\mathbf{0}$ & 2 & 2 & - & -1 & -1 & 0 & $\begin{array}{ll}0 & 0\end{array}$ & 0 & -2 & -2 & -2 & -2 & & & & 1 & 1 & 10 & 0 & 0 \\
\hline & 2 & 2 & 0 & & $2 \omega$ & $-2 \zeta$ & $-2 \omega$ & - & & 2 & 2 & 0 & 0 & $-2 \zeta$ & $2 \omega$ & - & & $-2 \omega$ & 0 & $\begin{array}{ll}0 & 0\end{array}$ & 0 & $2 \omega$ & $-2 \zeta$ & $\omega$ & $-2 \zeta$ & -1 & $\zeta$ & $-2 \omega$ & & -1 & $\begin{array}{ll}-2 \omega & 0\end{array}$ & $\begin{array}{ll}0 & 0\end{array}$ & 0 \\
\hline & 2 & 2 & 0 & & $-2 \zeta$ & $2 \omega$ & $\zeta$ & - & $-2 \omega$ & 2 & 2 & 0 & 0 & $2 \omega$ & $-2 \zeta$ & - & $-2 \omega$ & $\zeta$ & 0 & $\begin{array}{ll}0 & 0\end{array}$ & 0 & -2 & $2 \omega$ & & $2 \omega$ & -1 & $-2 \omega$ & $\zeta$ & $-2 \omega$ & -1 & $\zeta 0$ & & \\
\hline$x$ & 2 & 2 & 0 & & $-2 \zeta$ & $2 \omega$ & & - & $-2 \omega$ & -2 & -2 & 0 & 0 & $2 \omega$ & $-2 \zeta$ & -1 & $-2 \omega$ & $\zeta$ & 0 & $\begin{array}{ll}0 & 0\end{array}$ & 0 & $2 \zeta$ & $-2 \zeta$ & $2 \zeta$ & $-2 \zeta$ & 1 & $\omega$ & $-\zeta$ & $\omega$ & 1 & $-\zeta 0$ & 0 & 0 \\
\hline$x$ & 2 & 2 & 0 & 0 & $2 \omega$ & $-2 \zeta$ & $-2 \omega$ & & $\zeta$ & -2 & -2 & 0 & 0 & $-2 \zeta$ & $2 \omega$ & -1 & $\zeta$ & $-2 \omega$ & 0 & $\begin{array}{ll}0 & 0\end{array}$ & 0 & $-2 \zeta$ & $2 \zeta$ & $-2 \zeta$ & $2 \zeta$ & & & $\omega$ & $-\zeta$ & 1 & $\omega 0$ & 0 & 0 \\
\hline & 2 & -2 & o & o & 2 & 2 & -1 & -1 & -1 & $2 \mathbf{i}$ & $-2 \mathrm{i}$ & o & $\mathbf{0}$ & -2 & -2 & & & 1 & 0 & $\begin{array}{lll}0 & 0\end{array}$ & 0 & $-2 \mathbf{i}$ & $-2 \mathbf{i}$ & $2 \mathbf{i}$ & $2 \mathbf{i}$ & $\mathbf{i}-\mathbf{i}$ & $-\mathbf{i}$ & $-\mathbf{i}$ & $\mathbf{i}$ & i & i 0 & & 0 \\
\hline$\chi_{8}$ & 2 & -2 & 0 & 0 & 2 & 2 & -1 & & -1 & $-2 \mathbf{i}$ & $2 \mathbf{i}$ & o & o & -2 & -2 & & 1 & 1 & 0 & $\begin{array}{ll}0 & 0\end{array}$ & 0 & $2 \mathbf{i}$ & $2 \mathbf{i}$ & $-2 \mathbf{i}$ & $-2 \mathbf{i}$ & i i & i & & $-\mathbf{i}$ & $-\mathbf{i}$ & $\begin{array}{ll}-\mathbf{i} & 0\end{array}$ & 00 & 00 \\
\hline$\chi_{9}$ & 2 & -2 & 0 & 0 & $-2 \zeta$ & $2 \omega$ & & -1 & $-2 \omega$ & $2 i$ & $-2 i$ & 0 & 0 & $-2 \zeta$ & $2 \zeta$ & & $\omega$ & $-\zeta$ & 0 & $\begin{array}{ll}0 & 0\end{array}$ & 0 & $2 \eta^{5}$ & $2 \eta$ & $-2 \eta^{5}$ & $-2 \eta$ & $-i$ & & $\eta^{5}$ & $-\eta$ & $i$ & $\begin{array}{lll}-\eta^{5} & 0\end{array}$ & & \\
\hline$X 1$ & 2 & -2 & 0 & 0 & $2 \omega$ & $-2 \zeta$ & $-2 \omega$ & -1 & $\zeta$ & $2 i$ & $-2 i$ & 0 & 0 & $2 \zeta$ & $-2 \zeta$ & & $-\zeta$ & $\omega$ & 0 & $\begin{array}{ll}0 & 0\end{array}$ & 0 & $2 \eta$ & $2 \eta^{5}$ & $-2 \eta$ & $-2 \eta^{5}$ & $5-i$ & $\eta^{5}$ & & $-\eta^{5}$ & $i$ & $-\eta$ & & \\
\hline$\lambda 1$ & 2 & -2 & & & $-2 \zeta$ & $2 \omega$ & & -1 & $-2 \omega$ & $-2 i$ & $2 i$ & & & $-2 \zeta$ & $2 \zeta$ & & $\omega$ & $-\zeta$ & & $\begin{array}{ll}0 & 0\end{array}$ & 0 & $-2 \eta^{5}$ & $-2 \eta$ & $2 \eta^{5}$ & $2 \eta$ & $\eta \quad i$ & $-\eta$ & $-\eta^{5}$ & $\eta$ & $-i$ & $\eta^{5} 0$ & 0 & 0 \\
\hline & 2 & -2 & 0 & & $2 \omega$ & $-2 \zeta$ & $-2 \omega$ & -1 & & $-2 i$ & $2 i$ & 0 & 0 & $2 \zeta$ & $-2 \zeta$ & 1 & $-\zeta$ & $\omega$ & 0 & $\begin{array}{ll}0 & 0\end{array}$ & 0 & $-2 \eta$ & $-2 \eta^{5}$ & $2 \eta$ & $2 \eta^{5}$ & $i$ & $-\eta^{5}$ & $-\eta$ & $\eta^{5}$ & $-i$ & $\eta 0$ & $\begin{array}{lll}0 & 0 \\
\end{array}$ & $\begin{array}{ll}0 & 0 \\
0\end{array}$ \\
\hline
\end{tabular}


Furthermore, since $\beta \leq \alpha$ and $\delta \leq \gamma$, we have

$$
\begin{aligned}
& s=p^{\beta+\delta-\gamma} \leq p^{\alpha}, \\
& t=p^{\beta+\delta-\gamma} \leq p^{\beta} .
\end{aligned}
$$

Therefore, $a^{p^{\alpha}}=b^{p^{\beta}} \in\left\langle a^{s}\right\rangle \cap\left\langle b^{t}\right\rangle$. But since $a^{p^{\alpha}}$ is the smallest power of $a$ lying in the subgroup $\langle b\rangle$, we conclude that $\left\langle a^{p^{\alpha}}\right\rangle \cap\left\langle b^{p^{\beta}}\right\rangle$ is generated by $a^{p^{\alpha}}$, which has order $p^{\alpha+\delta} / p^{\alpha}=p^{\delta}$. Thus

$$
|Z(G)|=\frac{\left|\left\langle a^{s}\right\rangle\right| \cdot\left|\left\langle b^{t}\right\rangle\right|}{\left|\left\langle a^{s}\right\rangle \cap\left\langle b^{t}\right\rangle\right|}=\frac{\left|a^{s}\right| \cdot\left|b^{t}\right|}{\left|a^{p^{\alpha}}\right|}=\frac{p^{\alpha-\beta+\gamma} \cdot p^{\gamma}}{p^{\delta}}=p^{\alpha-\beta+2 \gamma-\delta} .
$$

Since $a^{s}$ and $b^{t}$ generate the abelian group $Z(G)$, we see that the exponent of $Z(G)$ is

$$
\exp Z(G)=\max \left(\left|a^{s}\right|,\left|b^{t}\right|\right)=\max \left(p^{\alpha-\beta+\gamma}, p^{\gamma}\right)=p^{\alpha-\beta+\gamma} .
$$

Therefore,

$$
Z(G) \cong \mathcal{C}_{\exp Z(G)} \times \mathcal{C}_{|Z(G)| / \exp Z(G)}=\mathcal{C}_{p^{\alpha-\beta+\gamma}} \times \mathcal{C}_{p^{\gamma-\delta}} .
$$

Since $\alpha-\beta+\gamma \geq \gamma-\delta$, we can then determine $\beta$ and $\delta$, and hence $G$.

Since nilpotent groups are direct products of their Sylow $p$-subgroups, we have

COROLlary 3.2. Any two nilpotent metacyclic groups of odd order having the same character tables are isomorphic.

We now deal with the case of 2-groups. We prove that any two metacyclic 2-groups which are not dihedral and generalized quaternion, respectively, can be distinguished by their character tables.

We will make use of the following characterization of metacyclic 2-groups by Hempel [6].

THEOREM 3.3. Every metacyclic 2-group has one of the following eight types of presentation, in which the parameters $r, s, t, u, v$ and $w$ are nonnegative integers:

(i) $\left\langle a \mid a^{2^{r}}=1\right\rangle$ with $r \geq 0$,

(ii) $\left\langle a, b \mid a^{2^{r}}=b^{2}=1, b^{a}=b\right\rangle$ with $r \geq 1$,

(iii) $\left\langle a, b \mid a^{2}=b^{2^{r}}=1, b^{a}=b^{-1}\right\rangle$ with $r \geq 2$,

(iv) $\left\langle a, b \mid a^{2}=b^{2^{r}}, b^{2^{r+1}}=1, b^{a}=b^{-1}\right\rangle$ with $r \geq 1$,

(v) $\left\langle a, b \mid a^{2}=b^{2^{r+1}}=1, b^{a}=b^{1+2^{r}}\right\rangle$ with $r \geq 2$,

(vi) $\left\langle a, b \mid a^{2}=b^{2^{r+1}}=1, b^{a}=b^{-1+2^{r}}\right\rangle$ with $r \geq 2$,

(vii) $\left\langle a, b \mid a^{2^{r}}=b^{2^{s}}, b^{2^{s+t}}=1, b^{a}=b^{1+2^{u}}\right\rangle$ with $r \geq s \geq u \geq 2$ and $u \geq t$,

(viii) $\left\langle a, b \mid a^{2^{r+s+t}}=b^{2^{r+s+u+v}}, b^{2^{r+s+u+v+w}}=1, b^{a}=b^{-1+2^{r+u}}\right\rangle$ where $r \geq 2, v \leq r, w \leq 1$, $s u=t u=t v=0$, and if $v \geq r-1$, then $w=0$.

Groups of different types or of the same type but with different parameters are not isomorphic.

Groups of type (i) and (ii) are abelian, and so are determined by their character tables. Types (iii) and (iv) are dihedral and generalized quaternion, respectively, and we have already seen that they have the same character table.

We will assume that $|G|>8$.

We note that groups of types (iii), (v), (vi) are split metacyclic and so no nonisomorphic pair of these groups have the same character tables by Theorem 1.1. 
The following table gives the orders of $G$ and isomorphism type of $G / G^{\prime}$ for groups of different types in terms of the parameters given in the characterization above.

\begin{tabular}{r|ll} 
type & $|G|$ & $G / G^{\prime}$ \\
\hline (iii), (iv) & $2^{r+1}$ & $\mathcal{C}_{2} \times \mathcal{C}_{2}$ \\
(v) & $2^{r+2}$ & $\mathcal{C}_{2} \times \mathcal{C}_{2^{r}}$ \\
(vi) & $2^{r+2}$ & $\mathcal{C}_{2} \times \mathcal{C}_{2}$ \\
(vii) & $2^{r+s+t}$ & $\mathcal{C}_{2^{r}} \times \mathcal{C}_{2^{u}}$ \\
(viii) & $2^{2 r+2 s+t+u+v+w}$ & $\mathcal{C}_{2} \times \mathcal{C}_{2^{r+s+t}}$
\end{tabular}

The information in this table follows immediately from the presentations.

Groups of type (vii) are distinguished from all other types by $G / G^{\prime}$, since $r, u \geq 2$. Groups of type (v) have $\left|G^{\prime}\right|=2$, which distinguishes them from the remaining types (iii), (iv), (vi), and (viii). Groups of type (viii) have $G / G^{\prime} \cong \mathcal{C}_{2} \times \mathcal{C}_{2^{r+s+t}}$. Since $r \geq 2$, this distinguishes type (viii) from types (iii), (iv) and (vi).

It remains to show that two groups of type (vii) or two groups of type (viii), with different parameters, cannot have the same character table.

Case (vii). We note that the proof of this case is nearly identical to the proof for odd $p$. The difference is that it appeals to the half of Lemma 3.1 which deals with $p=2$. Suppose $G$ is of type (vii), so that

$$
G=\left\langle a, b \mid a^{2^{r}}=b^{2^{s}}, b^{2^{s+t}}=1, b^{a}=b^{1+2^{u}}\right\rangle,
$$

where $r \geq s \geq u \geq 2$ and $u \geq t$. From the character table, we can determine $G / G^{\prime} \cong$ $\mathcal{C}_{2^{r}} \times \mathcal{C}_{2^{u}}$. Since $r \geq u$, we know both $r$ and $u$. From Lemma 2.2, we know that $Z(G)=$ $\left\langle a^{\alpha}, b^{\beta}\right\rangle$, where

$$
\begin{aligned}
& \alpha=\operatorname{ord}_{|b|}\left(1+2^{u}\right)=\operatorname{ord}_{2^{s+t}}\left(1+2^{u}\right), \\
& \beta=\frac{|b|}{\operatorname{gcd}\left(|b|, 2^{u}\right)}=\frac{2^{s+t}}{\operatorname{gcd}\left(2^{s+t}, 2^{u}\right)}=2^{s+t-u},
\end{aligned}
$$

where for the last equality we used the fact that $s+t \geq u$. Since $s, t, u$ satisfy $s+t \geq$ $u \geq 2$ and $u+2 \geq 4$, Lemma 3.1 implies that $\left(1+2^{u}\right)^{2^{j}} \equiv 1 \bmod 2^{s+t}$ if and only if $j \geq s+t-u$, and so $\alpha=\operatorname{ord}_{2^{s+t}}\left(1+2^{u}\right)=2^{s+t-u}$. Since $|a|=2^{r+t}$ and $|b|=2^{s+t}$, we have

$$
\left|a^{\alpha}\right|=2^{r+t} / \alpha=2^{r+u-s} \text {, and }\left|b^{\beta}\right|=2^{s+t} / \beta=2^{u} .
$$

Furthermore, since $r \geq s$ and $u \geq t$, we have

$$
\alpha=2^{s+t-u} \leq 2^{r} \text {, and } \beta=2^{s+t-u} \leq 2^{s} .
$$

Therefore, $a^{2^{r}}=b^{2^{s}} \in\left\langle a^{\alpha}\right\rangle \cap\left\langle b^{\beta}\right\rangle$. Since $a^{2^{r}}$ is the smallest power of $a$ lying in the subgroup $\langle b\rangle$, we conclude that $\left\langle a^{\alpha}\right\rangle \cap\left\langle b^{\beta}\right\rangle$ is generated by $a^{2^{r}}$, which has order $2^{r+t} / 2^{r}=2^{t}$. Thus

$$
|Z(G)|=\frac{\left|\left\langle a^{\alpha}\right\rangle\right| \cdot\left|\left\langle b^{\beta}\right\rangle\right|}{\left|\left\langle a^{\alpha}\right\rangle \cap\left\langle b^{\beta}\right\rangle\right|}=\frac{\left|a^{\alpha}\right| \cdot\left|b^{\beta}\right|}{\left|a^{2^{r}}\right|}=\frac{2^{r-s+u} \cdot 2^{u}}{2^{t}}=2^{r-s-t+2 u} .
$$


Since $\left|a^{\alpha}\right|=2^{r-s+u}>2^{u}=\left|b^{\beta}\right|$, we conclude that the exponent of $Z(G)$ is $\exp Z(G)=$ $2^{r-s+u}$. Thus

$$
Z(G) \cong \mathcal{C}_{\exp Z(G)} \times \mathcal{C}_{|Z(G)| / \exp Z(G)}=\mathcal{C}_{2^{r-s+u}} \times \mathcal{C}_{2^{u-t}} .
$$

Since $r-s+u \geq u-t$ and we know $r, u$, we can determine $s$ and $t$, and hence $G$.

Case (viii). Here we need Corollary 4.5 [6]:

LEMmA 3.4. If $G=\left\langle a, b \mid a^{2^{k}}=b^{2^{l}}, b^{2^{m}}=1, b^{a}=b^{-1+2^{n}}\right\rangle$, where $m-1 \leq l \leq m$, $2 \leq n \leq m$, and $m-n \leq k$, then

$$
Z(G)= \begin{cases}\left\langle a^{2}, b^{2^{m-1}}\right\rangle & \text { if } m=n, \\ \left\langle a^{2^{m-n}}, b^{2^{m-1}}\right\rangle & \text { otherwise. }\end{cases}
$$

A group of type (viii) is of the form

$$
G=\left\langle a, b \mid a^{2^{r+s+t}}=b^{2^{r+s+u+v}}, b^{2^{2+s+u+v+w}}=1, b^{a}=b^{-1+2^{r+u}}\right\rangle,
$$

where $r \geq 2, v \leq r, w \leq 1, s u=t u=t v=0$, and if $v \geq r-1$, then $w=0$.

From the above table we have:

$$
|G|=2^{2 r+2 s+t+u+v}, \quad G / G^{\prime} \cong \mathcal{C}_{2} \times \mathcal{C}_{2^{r+s+t}} .
$$

Thus, we know $2 r+2 s+t+u+v$ and $r+s+t$.

Lemma 3.5. The parameter $w$ can be determined from $Z(G)$. In particular: $w=1$ if and only if $Z(G)$ is cyclic of order greater than 2 .

Proof. Since $G$ satisfies the hypotheses of Lemma 3.4, we have

$$
Z(G)= \begin{cases}\left\langle a^{2}, b^{2^{++u-1}}\right\rangle & \text { if } s+v+w=0, \\ \left\langle a^{2^{s+v+w}}, b^{2^{++s+u+v+w-1}}\right\rangle & \text { otherwise. }\end{cases}
$$

We consider various cases.

Suppose first that $w=1$. Then, we are in the second of the two possibilities for $Z(G)$ in (3.1). We have $b^{2^{r+s+u+v+w-1}}=b^{r^{1+s+u+v}}=a^{2^{2+s+t}}$, and so

$$
Z(G)=\left\langle a^{2^{s+v+w}}, a^{2^{2+s+t}}\right\rangle=\left\langle a^{2^{\min (s+v+w, r+s+t)}}\right\rangle .
$$

Since $w=1$, we have $v<r-1$, and so $s+v+w<s+r \leq s+r+t$. Thus $Z(G)=$ $\left\langle a^{2^{s+v+w}}\right\rangle$, and so

$$
|Z(G)|=\left|a^{2 s+v+w}\right|=|a| / 2^{s+v+w}=2^{r+s+t+w} / 2^{s+v+w}=2^{r+t-v} .
$$

Since $v<r-1$, we have $r+t-v \geq 2$, and so $Z(G) \cong \mathcal{C}_{2^{r+t-v}}$ is cyclic of order $2^{r+t-v}>$ 2 .

Now suppose that $w=0$. Consider the situation $v=r \geq 2$. Then, we are again in the second case of (3.1):

$$
Z(G)=\left\langle a^{2^{s+v+w}}, b^{2^{r+s+u+v+w-1}}\right\rangle=\left\langle a^{2^{r+s}}, b^{2^{2+s+u+v-1}}\right\rangle .
$$


From the condition $t v=0$, we have $t=0$, and so $a^{2+s}=a^{2^{r+s+t}}=b^{2 r+s+u+v}$. Thus $Z(G)$ is generated by $b^{2+s+u+v-1}$, which has order

$$
|b| / 2^{r+s+u+v-1}=2^{r+s+u+v} / 2^{r+s+u+v-1}=2,
$$

so $Z(G) \cong \mathcal{C}_{2}$.

If $w=0$ and $v \neq r$, we show that $Z(G)$ is noncyclic.

If $s+v=0$, then $Z(G)=\left\langle a^{2}, b^{2^{r+u-1}}\right\rangle$. Now $\left|a^{2}\right|=2^{r+t} / 2=2^{r+t-1}$ and $\left|b^{2^{r+u-1}}\right|=$ $|b| / 2^{r+u-1}=2^{r+u} / 2^{r+u-1}=2$. Furthermore, since $G$ is split metacyclic, the intersection $\left\langle a^{2}\right\rangle \cap\left\langle b^{2^{r+u-1}}\right\rangle \subseteq\langle a\rangle \cap\langle b\rangle$ is trivial, and so

$$
Z(G)=\left\langle a^{2}\right\rangle \times\left\langle b^{2^{r+u-1}}\right\rangle \cong \mathcal{C}_{2^{r+t-1}} \times \mathcal{C}_{2} .
$$

Since, $r \geq 2$ we see that $Z(G)$ is non-cyclic.

If $s+v>0$, then $Z(G)=\left\langle a^{s^{s+v}}, b^{2^{r+s+u+v-1}}\right\rangle$. The generators have order

$$
\begin{aligned}
\left|a^{s+v}\right| & =|a| / 2^{s+v}=2^{r+s+t} / 2^{s+v}=2^{r+t-v}, \text { and } \\
\left|b^{2^{r+s+u+v-1}}\right| & =|b| / 2^{r+s+u+v-1}=2^{r+s+u+v} / 2^{r+s+u+v-1}=2 .
\end{aligned}
$$

Again, since $G$ is split metacyclic, the intersection $\left\langle a^{2 s+v}\right\rangle \cap\left\langle b^{r^{2+s+u+v-1}}\right\rangle \subseteq\langle a\rangle \cap\langle b\rangle$ is trivial, and so

$$
Z(G)=\left\langle a^{2 s+v}\right\rangle \times\left\langle b^{2^{+s+u+v-1}}\right\rangle \cong \mathcal{C}_{2^{r+t-v}} \times \mathcal{C}_{2} .
$$

Here, we note that $r+t-v>0$ since we are assuming the conditions $r \geq v$ and $r \neq v$.

Thus, $w$ can be determined by the isomorphism type of $Z(G)$ in all cases.

If $w=1$, then we consider the quotient

$$
G /\left\langle b^{2^{r+s+u+v}}\right\rangle \cong\left\langle a, b \mid a^{2^{r+s+t}}=b^{2^{r+s+u+v}}=1, b^{a}=b^{-1+2^{r+u}}\right\rangle,
$$

by the unique central subgroup of $G$ of order 2. This quotient is a group of type (viii) with the same $r, s, t, u, v$, but with $w=0$. Thus, we may reduce to the case where $w=0$. Then we have

$$
G=\left\langle a, b \mid a^{2^{r+s+t}}=b^{2^{r+s+u+v}}=1, b^{a}=b^{-1+2^{r+u}}\right\rangle,
$$

and we note that $G$ is split metacyclic. This case now follows from Theorem 1.1.

This completes the proof of Theorem 1.2.

4. $\{p, q\}$-groups. We first give a description of these groups according to the characterization given in [10]. Let $p, q$ be odd primes with $p$ dividing $q-1$, let $\mu$ be the largest integer such that $p^{\mu}$ divides $q-1$, and let $\alpha, \beta, \gamma, \delta, \kappa$ be nonnegative integers satisfying

(i) $\alpha \leq \beta, \gamma \geq \beta, \beta+\delta \geq \gamma \geq \delta$,

(ii) $\delta \geq 1$ or $\beta=0$,

(iii) $1 \leq \kappa \leq \min (\alpha, \mu)$,

(iv) $\beta \geq \delta$ or $\alpha-\kappa<\beta$. 
Let $\epsilon, \zeta, \eta$ be nonnegative integers with $\epsilon+\eta \geq \zeta \geq \eta>0$ and let $\theta$ be a primitive $p^{\kappa}$ th root of unity $\bmod q^{\zeta}$. Then it is proven in [10] that the group $G$ generated by $x, y, u, v$ and given with the relations

$$
\begin{array}{lll}
x^{p^{\alpha}}=y^{p^{\beta}}, & \\
y^{x}=y^{1+p^{\delta}}, & y^{p^{\gamma}}=1, & \\
u^{x}=u, & u^{y}=u, & u^{q^{\epsilon}}=1, \\
v^{x}=v^{\theta}, & v^{y}=v, & v^{u}=v^{1+q^{\eta}},
\end{array}
$$

is a presentation of a non-nilpotent metacyclic group of order $p^{\alpha+\gamma} q^{\epsilon+\zeta}$, and that every non-nilpotent metacyclic $\{p, q\}$-group has a presentation of this form. Moreover, [10], $\theta$ can be chosen in a manner depending on $\alpha, \beta, \gamma, \delta, \kappa$ so that the groups given by presentations as above form a complete and irredundant set of representatives of the isomorphism types of non-nilpotent metacyclic $\{p, q\}$-groups. Note that the elements $x u$ and $y v$ are generators giving a metacyclic presentation for $G$, and that it is not clear from the above presentation that $G$ will be split metacyclic unless $\alpha \beta=0$.

We note that the cases where the $\{p, q\}$-group is nilpotent are covered by Corollary 3.2.

Proof of Theorem 1.3. So, let $G$ be a metacyclic $\{p, q\}$-group with a presentation as above, and where by [10] the parameters $\alpha, \beta, \gamma, \delta, \kappa, \epsilon, \zeta, \eta$ are invariants of $G$ which determine $G$ up to isomorphism. Let $P=\langle x, y\rangle$ and $Q=\langle u, v\rangle$. Then $Q$ is a normal Sylow $q$-subgroup, and so can be located by the character table. Since $G / Q \cong P$, we can determine the character table of $P$ from the character table of $G$. Since, $p$ is odd and $P$ is a metacyclic $p$-group with presentation

$$
P=\left\langle x, y \mid x^{p^{\alpha}}=y^{p^{\beta}}, y^{p^{\gamma}}=1, y^{x}=y^{1+p^{\delta}}\right\rangle,
$$

we can determine the parameters $\alpha, \beta, \gamma$, and $\delta$ using Theorem 1.2.

Lemma 5.6 of [10] states that $\kappa=\left|P: \mathbf{O}_{p}(G)\right|$, where $\mathbf{O}_{p}(G)$ is the unique largest normal $p$-subgroup of $G$. Since the orders of $P$ and of $\mathbf{O}_{p}(G)$ can be determined by the character table, this gives us $\kappa$.

Let $X=\langle x\rangle$ and $V=\langle v\rangle$. Note that since $P$ centralizes $u$ and $y$ centralizes $v$, we have $G^{\prime} \cap Q=[P, Q]=[X, V]$. We can write $V=C_{V}(X) \times[X, V]$, but since $V$ is cyclic, one of these direct factors must be trivial. Since the action of $x$ on $v$ is nontrivial (since $G$ is not nilpotent), we conclude that $V=[X, V]$. Now $G^{\prime}$ can be located from the character table. In particular, since $G^{\prime}$ is cyclic, it has a unique subgroup of every order dividing $\left|G^{\prime}\right|$. These subgroups are normal in $G$, and so we can find the Sylow $q$-subgroup of $G^{\prime}$, which is $V$. Thus, we can determine $\zeta=|V|$ from the character table. From the order of $G$, which is equal to $p^{\alpha+\gamma} q^{\epsilon+\zeta}$, we can also determine $\epsilon+\zeta$, and hence $\epsilon$.

To find $\eta$, we examine $Q \cap Z(G)$. From the presentation of $G$, we see that $Q \cap$ $Z(G)=C_{Z(Q)}(x)$. Since

$$
Q=\left\langle u, v \mid u^{q^{\epsilon}}=v^{q^{\zeta}}=1, v^{u}=v^{1+q^{\eta}}\right\rangle
$$


we know from Lemma 2.2 that $Z(Q)=\left\langle u^{S}, v^{T}\right\rangle$, where

$$
\begin{aligned}
& S=\operatorname{ord}_{q^{\zeta}}\left(1+q^{\eta}\right), \\
& T=q^{\zeta} / \operatorname{gcd}\left(q^{\zeta}, q^{\eta}\right)=q^{\zeta-\eta} .
\end{aligned}
$$

Since $\zeta \geq \eta \geq 1$ and $\eta+q \geq 4$, Lemma 3.1 implies that $\left(1+q^{\eta}\right)^{q^{n}} \equiv 1 \bmod q^{\zeta}$ if and only if $n \geq \zeta-\eta$, and so $S=\operatorname{ord}_{q^{\zeta}}\left(1+q^{\eta}\right)=q^{\zeta-\eta}$. The above generators of $Z(Q)$ have orders

$$
\begin{aligned}
& \left|u^{S}\right|=|u| / S=q^{\epsilon} / q^{\zeta-\eta}=q^{\epsilon-\zeta+\eta}, \\
& \left|v^{T}\right|=|v| / T=q^{\zeta} / q^{\zeta-\eta}=q^{\eta} .
\end{aligned}
$$

Since $Q$ is split, it then follows that

$$
Z(Q)=\left\langle u^{S}, v^{T}\right\rangle \cong \mathcal{C}_{q^{\epsilon-\zeta+\eta}} \times \mathcal{C}_{q^{\eta}}
$$

Since $u^{x}=u$, we have

$$
Q \cap Z(G)=C_{Z(Q)}(x) \cong \mathcal{C}_{q^{\epsilon-\zeta+\eta}} \times \mathcal{C}_{\left\langle v^{T}\right\rangle}(x) .
$$

Since, this last factor is cyclic of order at most $q^{\eta} \leq q^{\epsilon-\zeta+\eta}$, we conclude that the Sylow $q$-subgroup of $Z(G)$ has exponent $q^{\epsilon-\zeta+\eta}$. Therefore, we can determine $\epsilon-\zeta+\eta$, and hence $\eta$, from the character table. This gives all the parameters for $G$.

Acknowledgement. We would like to thank Darrin Doud for valuable conversations regarding this paper. All computations made in the preparation of this paper were accomplished using Magma [8].

\section{REFERENCES}

1. B. G. Basmaji, On the isomorphisms of two metacyclic groups. Proc. Am. Math. Soc. 22 (1969), 175-182.

2. C. W. Curtis, Pioneers of representation theory: Frobenius, Burnside, Schur, and Brauer, History of Mathematics, vol. 15 (American Mathematical Society, Providence, RI; London Mathematical Society, London, 1999), 287.

3. C. W. Curtis and I. Reiner, Representation theory of finite groups and associative algebras, Reprint of the 1962 original. (AMS Chelsea Publishing, Providence, RI, USA, 2006).

4. W. Feit, Characters of finite groups (W. A. Benjamin, New York, USA, 1967).

5. W. Fulton and J. Harris, Representation theory. A first course. Graduate Texts in Mathematics. Readings in Mathematics, vol. 129 (Springer-Verlag, New York, USA, 1991).

6. C. E. Hempel, Metacyclic groups, Comm. Algebra 28(8) (2000), 3865-3897.

7. I. M. Isaacs, Finite group theory, Graduate Studies in Mathematics, vol. 92 (American Mathematical Society, Providence, RI, USA, 2008).

8. W. Bosma and J. Cannon, MAGMA (University of Sydney, 1994).

9. H. J. Munkholm, Induced monomial representations, Young elements, and metacyclic groups, Proc. Am. Math. Soc. 19 (1968), 453-458.

10. H.-S. Sim, Metacyclic groups of odd order, Proc. London Math. Soc. 69(3) (1994), 47-71.

11. H. J. Zassenhaus, The theory of groups, Reprint of the second (1958) ed. (Dover Publications, Inc., Mineola, New York, USA, 1999). 